\title{
AVALIAÇÃO DO TEOR DE METAIS EM SEDIMENTO DO RIO BETARI NO PARQUE ESTADUAL TURÍSTICO DO ALTO RIBEIRA - PETAR, SÃO PAULO, BRASIL
}

\author{
Jussara Aparecida Oliveira Cotta*, Maria Olímpia Oliveira Rezende e Mônica R. Piovani \\ Instituto de Química de São Carlos, Universidade de São Paulo, Av. Trabalhador São Carlense, 400, 13560-970 São Carlos- SP \\ Recebido em 14/10/04; aceito em 4/5/05; publicado na web em 10/8/05
}

\begin{abstract}
EVALUATION OF METAL CONTENT IN SEDIMENTS OF THE BETARI RIVER IN THE PARQUE ESTADUAL TURÍSTICO DO ALTO RIBEIRA -PETAR-, SÃO PAULO, BRAZIL. The subsoil of the Vale do Ribeira was the focus of mining industries for the exploration of lead, mainly inside the park - PETAR. Despite the fact that the exploration has ended, the environmental effects of those activities are still present, due to great amounts of heavy metals that are leached. Concentrations of pseudo-total and bioavailable metals were determined in sediment samples of the Betari River, using atomic absorption spectrometry. The results demonstrated that the sediments are contaminated by $\mathrm{Pb}, \mathrm{Zn}$ and $\mathrm{Cu}$. The findings can contribute to an efficient and environmentally and economically adequate management of the park, for the conservation and the protection of the area.
\end{abstract}

Keywords: river sediment; trace metals; sustainable development.

\section{INTRODUÇÃO}

O crescimento industrial desordenado gera como consequiência uma grande liberação de compostos indesejáveis ao meio ambiente, causando danos à vida animal e vegetal. Desta forma, apesar dos bens minerais contribuírem para o desenvolvimento industrial, são, também, fontes poluidoras quando lançados em grande quantidade ao meio ambiente ${ }^{1}$.

O lançamento dos efluentes industriais e domésticos, o intemperismo e outros processos pedogênicos que sobre a rocha matriz geram os elementos-traço podem propiciar um perfil completamente diferente ao da dinâmica de sedimentação encontrada nos ambientes naturais não perturbados ${ }^{2}$. Mas essas não são as únicas fontes que geram esse tipo de elementos os quais podem, ainda, ser incorporados aos ambientes naturais por microrganismos, decomposição de restos de animais e fontes antropogênicas ${ }^{3}$.

Os sedimentos desempenham um papel importante no tocante ao destino de xenobióticos em ambientes aquáticos, pois refletem a quantidade corrente do sistema aquático e podem ser usados para detectar a presença de contaminantes que não permanecem solúveis após seu lançamento em águas superficiais ${ }^{4}$. Os sedimentos são considerados de grande importância na avaliação do nível de contaminação dos ecossistemas aquáticos, devido não só a sua capacidade em acumular elementos-traço mas, também, por serem reconhecidos como transportadores e possíveis fontes de contaminação, já que tal compartimento ambiental pode liberar espécies contaminantes ${ }^{5,6}$. Tais espécies são geralmente liberadas do leito do sedimento devido a alterações nas condições ambientais e físico-químicas ( $\mathrm{pH}$, potencial redox e ação microbiana, entre outras), podendo contaminar a água e outros sistemas ambientais, afetando a qualidade da água, levando à bioacumulação e transferência na cadeia trófica ${ }^{5,7}$.

Os metais, principalmente os metais pesados, em grande concentração, merecem maior preocupação, principalmente por serem não degradáveis, permanecendo por longos períodos no ambiente, principalmente nos sedimentos. Nos últimos anos, tem aumentado

\footnotetext{
*e-mail: japcotta@iqsc.usp.br
}

a investigação sobre metais presentes em sedimentos, não mais como um reservatório ou ambiente de deposição de espécies químicas, mas como um compartimento aquático ativo que desempenha um papel fundamental na redistribuição dessas espécies à biota aquática.

Os fenômenos de acúmulo e de redisposição de espécies nos sedimentos os qualificam como de extrema importância em estudos de impacto ambiental, pois registram em caráter mais permanente os efeitos de contaminação ${ }^{4}$. Assim sendo, a determinação de metais-traço em sedimentos permite detectar o grau de contaminação a que a água e os organismos bentônicos estão sujeitos ${ }^{8}$.

A ação química dos metais pesados tem despertado grande interesse ambiental. Isso se deve, em parte, ao fato de não possuírem caráter de biodegradação, o que determina que permaneçam em ciclos biogeoquímicos globais nos quais as águas naturais são seus principais meio de condução, podendo-se acumular na biota aquática em níveis elevados. Vários trabalhos têm voltado seu interesse para a quantificação de poluição por metais pesados em sedimentos, reunindo dados sobre o impacto ambiental e suas complexas relações com as atividades econômicas ${ }^{6,9-13}$.

\section{DESCRIÇÃO DA ÁREA DE ESTUDO}

O Parque Estadual Turístico do Alto Ribeira - PETAR, criado em 1958 e administrado pelo Instituto Florestal/Secretaria do Meio Ambiente, representa uma unidade de conservação com 35.102,8 ha localizada na margem esquerda do alto curso do Rio Ribeira, no Vale do Ribeira, sul do estado de São Paulo, e abrange parte dos municípios de Iporanga e Apiaí14.

A região com mais de 1200 ha de floresta é uma das últimas áreas de Mata Atlântica preservadas no estado e considerada como a região de maior concentração de cavernas do Brasil, além de apresentar cachoeiras, rios, montanhas, vales e uma diversidade enorme de fauna e flora. Juntamente com essa diversidade ambiental há elementos culturais de grande interesse. O Vale do Ribeira apresenta os menores valores de desenvolvimento social do estado de São Paulo e sua população não dispõe de alternativas econômicas para um desenvolvimento sustentável que permita o uso racional de seus recursos naturais e culturais. 
Aproximadamente 29\% da área ocupada do PETAR são utilizadas por atividades consideradas impróprias para a preservação desse patrimônio público, representando um risco permanente para os ecossistemas da região. Essas atividades consistem basicamente na agricultura, exploração mineral, descarga de esgoto doméstico não tratado e expansão populacional ${ }^{15}$.

Vários trabalhos têm avaliado o quanto tais atividades têm afetado não só o ambiente no PETAR ${ }^{16-20}$, mas também o Vale do Ribeira $^{21-24}$, que sofre constantes riscos ecológicos. Segundo a Superintendência do Desenvolvimento do Litoral Paulista (SUDELPA), o subsolo do PETAR foi sempre foco de interesse da indústria mineradora, sendo que $80 \%$ da área do PETAR foi considerada de interesse para mineração. A primeira jazida explorada economicamente seria a mina Furnas, localizada dentro do próprio PETAR, manifestada em 1919, sendo o minério principal composto por galena argentífera, exportada para a Espanha. Em 1992, a mina foi fechada por dificuldades tecnológicas encontradas para beneficiamento de seu minério. O minério primário de Furnas é constituído, predominantemente, por galena, pirita e esfalerita, com participação de arsenopirita, calcopirita e estibinita ${ }^{21}$. A mina localiza-se às margens do ribeirão Furnas, afluente do alto curso do rio Betari que, por sua vez, é afluente da margem esquerda do rio Ribeira à altura da cidade de Iporanga - SP.

Melcher ${ }^{25}$ estimou que esta mina produziu, até 1968 , entre 7.000 e $8.000 \mathrm{t}$ de chumbo, representando $5-6 \%$ da produção total da região do vale do rio Ribeira de Iguape. Chumbo foi o metal mais extraído no Vale do Ribeira, principalmente nas municipalidades de Iporanga e Apiaí. Embora não haja mais a exploração do chumbo, os efeitos ambientais dessa atividade continuam presentes, via descarga dos efluentes e rochas com alto conteúdo de metais pesa$\operatorname{dos}^{26}$.

Alguns estudos têm reportado o impacto ambiental por metais provenientes de atividades de mineração ${ }^{27-30}$. Neste trabalho, os metais avaliados foram $\mathrm{Pb}$, devido aos problemas com a mineração, $\mathrm{Cd}, \mathrm{Cu}$ e $\mathrm{Zn}$ pois, segundo a CETESB, esses metais têm sido encontrados no PETAR ${ }^{26}$. Também foram avaliados $\mathrm{Al}, \mathrm{Cr}, \mathrm{Fe}, \mathrm{Mn}$ e Ni.

\section{PARTE EXPERIMENTAL}

\section{Coleta das amostras}

Para a coleta das amostras, realizada em janeiro de 2003, foram estabelecidos 6 pontos de amostragem. Os sedimentos depositados em ambientes de baixa energia, ou seja, em encostas de inundação, foram escolhidos como objeto de estudo. As condições morfológicas e hidrológicas em ambientes de encostas de inundação favorecem o acúmulo, durante o período de cheia, de sedimentos finos transportados em suspensão. A granulometria fina dos sedimentos normalmente encontrados nesses ambientes é indicada para estudos de avaliação do grau de contaminação por metais pesados, devido à elevada superfície específica. As amostras, coletadas com draga Vanveen, foram acondicionadas em sacos plásticos e congeladas a $4{ }^{\circ} \mathrm{C}$ até o processamento.

O mapa da região é mostrado na Figura 1, com a identificação dos pontos de amostragem. O ponto 1 foi estabelecido no rio Furnas, o 2 na confluência dos rios Furnas e Betari, e o 3 no rio Betari, localizado no bairro da Serra (bairro pertencente ao município de Iporanga e localizado nas limitações do PETAR). O ponto 4 situava-se no final do bairro da Serra, o 5 no rio Fria (antes da confluência) e o 6 no rio Betari próximo ao rio Ribeira. Destaca-se o ponto 1, por ser um rio afetado pelos rejeitos da mineradora de Furnas. O bairro da Serra foi escolhido por ficar na região limitrofe do PETAR e concentrar a densidade demográfica. Os diversos pontos de

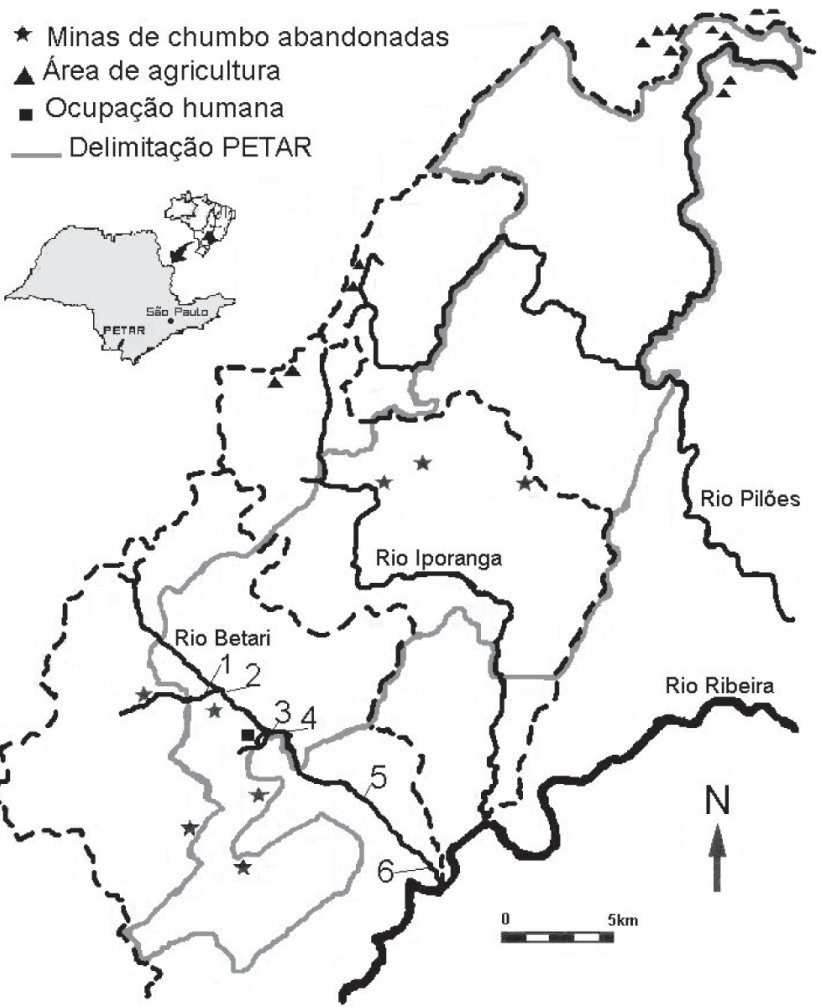

Figura 1. Mapa de localização da área de estudo ${ }^{16}$ e dos pontos de amostragem

amostragem foram distribuídos ao longo do rio Betari para permitir a avaliação da influência das atividades de mineração, a que se referem as concentrações dos metais.

A caracterização física e química dos sedimentos (Tabela 1) foi realizada por $\operatorname{Cotta}^{31}$ e as análises granulométricas foram realizadas seguindo a norma técnica NBR 7181/1982 ${ }^{32}$, pelo Laboratório de Estradas do Departamento de Transportes da Escola de Engenharia de São Carlos da Universidade de São Paulo.

\section{Reagentes e soluções}

Toda a vidraria foi limpa com detergente Extran (Merck), enxaguada com água desionizada, posteriormente submersa em solução de ácido nítrico a $10 \%$ (v/v) e mantida por uma noite nessas condições. Em seguida, o material foi enxaguado com água desionizada ${ }^{8}$.

Todas as soluções foram preparadas com reagentes de grau analítico (Merck) e água desionizada purificada através do sistema Milli-Q (Millipore) até alta pureza (resistividade $18 \mathrm{M} \Omega \mathrm{cm}^{-1}$ ). As soluções de referência, para obtenção das curvas analíticas para determinação dos metais nas amostras de sedimento, foram preparadas por diluição serial das soluções estoque de $1 \times 10^{3} \mathrm{mg} \mathrm{L}^{-1}$ de $\mathrm{Al}, \mathrm{Cr}, \mathrm{Fe}, \mathrm{Mn}, \mathrm{Ni}, \mathrm{Cd}, \mathrm{Cu}, \mathrm{Zn}$ e $\mathrm{Pb}$ em $\mathrm{HNO}_{3}$ 1,00 mol L-1 (v/v).

Os ácidos utilizados, $\mathrm{HNO}_{3}$ e $\mathrm{HCl}$, foram de procedência pa (Mallinckrodt).

\section{Preparo das amostras}

Para a determinação da capacidade de troca catiônica e os metais biodisponíveis, as amostras foram primeiramente peneiradas em malha de $250 \mu \mathrm{m}$ e colocadas em estufa a $50{ }^{\circ} \mathrm{C}$ por $24 \mathrm{~h}$. O sedimento seco foi triturado para homogeneização.

Para determinação de metais pseudo-totais a amostra, seca em 
Tabela 1. Caracterização física e química das amostras de sedimento

\begin{tabular}{|c|c|c|c|c|c|c|}
\hline Pontos de amostragem & 1 & 2 & 3 & 4 & 5 & 6 \\
\hline $\mathrm{pH}^{\mathrm{a}}$ & 6,99 & 6,02 & 7,30 & 6,21 & 6,21 & 6,94 \\
\hline Carbono orgânico $(\mathrm{g} / \mathrm{kg})^{\mathrm{b}}$ & 5,65 & 2,78 & 8,07 & 21,27 & 3,14 & 34,77 \\
\hline Umidade $(\%)^{\mathrm{c}}$ & 19,75 & 22,18 & 32,62 & 34,07 & 38,81 & 14,27 \\
\hline Teor de Matéria orgânica $(\%)^{\mathrm{d}}$ & 2,99 & 4,34 & 3,88 & 6,04 & 6,74 & 5,05 \\
\hline Nitrogênio total Kjeldahl $(\mathrm{g} / \mathrm{kg})^{\mathrm{e}}$ & 0,60 & 0,30 & 0,30 & 0,75 & 0,45 & 0,45 \\
\hline Fósforo $(\mathrm{g} / \mathrm{kg})^{\mathrm{e}}$ & 1,33 & 0,84 & 0,80 & 1,17 & 0,71 & 1,19 \\
\hline $\operatorname{Argila}(\%)^{\mathrm{f}}$ & 0 & 2 & 2,5 & 4 & 6 & 4 \\
\hline $\operatorname{Silte}(\%)^{\mathrm{f}}$ & 11,5 & 9 & 52,5 & 20 & 22,5 & 64 \\
\hline $\operatorname{Areia}(\%)^{\mathrm{f}}$ & 66,5 & 70 & 45 & 76 & 42,5 & 24 \\
\hline Pedregulho $(\%)^{\mathrm{f}}$ & 12 & 13 & 0 & 0 & 11 & 1 \\
\hline Agregado $(\%)^{\mathrm{f}}$ & 10 & 6 & 0 & 0 & 18 & 7 \\
\hline
\end{tabular}

adeterminação da atividade hidrogeniônica através de uma suspensão com $25,00 \mathrm{~mL}$ de solução de cloreto de cálcio $0,01 \mathrm{~mol} \mathrm{~L}^{-1} \mathrm{e} 10,00 \mathrm{~g}$ de cada amostra coletada; ${ }^{b}$ medidor de carbono total, modelo TOC-V ${ }_{\mathrm{CPH}}$, acoplado ao módulo de amostras sólidas, modelo SSM-5000A, marca Shimadzu, com detector de combustão; 'em estufa a $60-65$ e a $100-110{ }^{\circ} \mathrm{C}$; ${ }^{\mathrm{d}}$ Calcinação em mufla a $500{ }^{\circ} \mathrm{C}$ por $4 \mathrm{~h}$; ${ }^{\mathrm{e}}$ medida em espectrofotômetro, modelo DR/2500, marca HACH; fjogo de peneiras de números: 16, 40, 60, 100 e 230, as quais foram agitadas em mesa agitadora por $10 \mathrm{~min}$. As determinações da fração silte e argila foram feitas pelo método do densímetro, da norma técnica (NBR).

estufa a $50{ }^{\circ} \mathrm{C}$ por $24 \mathrm{~h}$, foi triturada e peneirada em peneira de nylon de $74 \mu \mathrm{m}$ de abertura de malha. uma vez que muitos estudos têm demostrado que as concentrações mais elevadas de metais ocorrem nas frações mais finas do sedimento ${ }^{33,34}$. As frações mais finas compreendem os principais carreadores dos componentes naturais e antropogênicos a partir da coluna d'água. Nas frações silte e areia fina, as concentrações de metais decrescem, porque elas são predominantemente formadas por compostos de quartzo com baixos teores de metais ${ }^{6}$.

\section{Instrumentação}

As determinações de metais totais e biodisponíveis foram realizadas por espectrometria de absorção atômica (EAA), em espectrômetro marca Hitachi, modelo Z-8100, equipado com polarizador Zeeman. O sistema de aquisição de dados foi gerenciado por microcomputador e, como fonte de radiação, foram empregadas lâmpadas de cátodo oco dos respectivos metais.

\section{Determinação da capacidade de troca catiônica}

Para determinação dos cátions metálicos totais trocáveis foram pesados 2,50 g de amostra, adicionando-se 25,00 $\mathrm{mL}$ de $\mathrm{CH}_{3} \mathrm{COOH} 1,00 \mathrm{~mol} \mathrm{~L}^{-1}$. A suspensão foi agitada por $1 \mathrm{~h}$ e, então, foi determinado o $\mathrm{pH}$, assim como o $\mathrm{pH}$ da solução de $\mathrm{CH}_{3} \mathrm{COOH}^{35}$. Pela equação a seguir, determinaram-se os cátions metálicos totais trocáveis.

Cátions metálicos trocáveis $\left(\mathrm{cmol}_{\mathrm{c}} / \mathrm{kg}\right)=\left[\mathrm{pH}_{1}-\mathrm{pH}_{2}\right]$ x 22

em que: $\mathrm{pH}_{1}=\mathrm{pH}$ da suspensão; $\mathrm{pH}_{2}=\mathrm{pH}$ da solução de $\mathrm{CH}_{3} \mathrm{COOH}$.

Para determinação da acidez trocável foram colocados 5,00 g de cada amostra em erlenmeyer de $125,00 \mathrm{~mL}$ e adicionados 50,00 $\mathrm{mL}$ de $\mathrm{KCl} 1,00 \mathrm{~mol} \mathrm{~L}^{-1}$. Agitou-se a mistura manualmente algumas vezes, deixou-se em repouso durante $30 \mathrm{~min}$ e filtrou-se em papel de filtro, adicionando-se duas porções de $10,00 \mathrm{~mL}$ de $\mathrm{KCl}$ $1,00 \mathrm{~mol} \mathrm{~L}^{-1}$. Adicionaram-se ao filtrado 6 gotas de fenolftaleína a $0,10 \%(\mathrm{~m} / \mathrm{v})$ e titulou-se com $\mathrm{NaOH} 0,01 \mathrm{~mol} \mathrm{~L}^{-1}$.

Pela equação a seguir, determinou-se a acidez trocável.

Acidez trocável $\left(\mathrm{cmol}_{\mathrm{c}} / \mathrm{kg}\right)=(\mathrm{V} \times \mathrm{C} \times 100) / \mathrm{m}$ onde: $\mathrm{V}=$ volume $(\mathrm{em} \mathrm{mL}$ ) de $\mathrm{NaOH}$ gastos na titulação; $\mathrm{C}=$ concentração (em mol/L) da solução de $\mathrm{NaOH} ; \mathrm{m}=$ massa (em g) da $\operatorname{amostra}^{36,37}$.

\section{Determinação de metais biodisponíveis e pseudo-totais}

Para determinação da concentração dos metais biodisponíveis pesou-se cerca de $1,00 \mathrm{~g}$ da amostra em um erlenmeyer e adicionaram-se $25,00 \mathrm{~mL}$ de $\mathrm{HCl} 0,10 \mathrm{~mol} \mathrm{~L}^{-1}$, submetendo-se a mistura à agitação em um agitador horizontal, a $200 \mathrm{rpm}$, por $2 \mathrm{~h}$. A suspensão foi, então, filtrada por gravidade e o filtrado armazenado a $4{ }^{\circ} \mathrm{C}{ }^{38}$.

Para determinação de metais pseudo-totais, aproximadamente $0,50 \mathrm{~g}$ de cada amostra foi colocada nos tubos do bloco digestor, aos quais foram adicionados $15,00 \mathrm{~mL}$ de $\mathrm{HNO}_{3}$ concentrado p.a. Após $12 \mathrm{~h}$ em repouso, aqueceu-se até $160{ }^{\circ} \mathrm{C}$, permanecendo nesta temperatura por $4 \mathrm{~h}$. Foram, então, adicionados $8,00 \mathrm{~mL}$ de peróxido de hidrogênio $30 \%(\mathrm{v} / \mathrm{v})$, deixando-se à temperatura de $160{ }^{\circ} \mathrm{C}$ por mais $30 \mathrm{~min}$. As amostras foram transferidas para um balão volumétrico de $100,00 \mathrm{~mL}$, completando-se o volume e removendo-se a parte não digerida por filtração. Esse procedimento segue o método 3050 da Environmental Protection Agengy (EPA) ${ }^{39}$.

A qualidade dos resultados analíticos foi acompanhada com o uso do branco analítico e de determinações em triplicata, distribuídas aleatoriamente. Em alguns casos foram necessárias diluições das amostras para as determinações de $\mathrm{Fe}, \mathrm{Mn}, \mathrm{Pb}, \mathrm{Zn}$ e $\mathrm{Al}$ por EAA, pois estas se encontravam em concentrações mais elevadas que as utilizadas na curva analítica.

\section{RESULTADOS E DISCUSSÃO}

\section{Determinação da capacidade de troca catiônica efetiva}

Um cátion raramente existe na forma trocável e, em geral, cátions disponíveis podem ser considerados como cátions trocáveis. A quantidade total de cátions trocáveis que pode ser retida em um material é conhecida como capacidade de troca cationnica (CTC). Entende-se por CTC efetiva a capacidade de troca de cátions do sedimento ou a capacidade do sedimento em reter cátions, em um valor de $\mathrm{pH}$ próximo ao seu valor natura ${ }^{40}$.

A acidez $(\mathrm{H}+\mathrm{Al})$ liberada pela reação com solução não tamponada de $\mathrm{KCl}$ pode ser designada como acidez real e é utilizada para determinar o que se denomina de CTC efetiva, isto é, a soma 
Tabela 2. Valores da CTC efetiva nas amostras de sedimento

\begin{tabular}{cc}
\hline Pontos de amostragem & CTC efetiva $\left(\mathrm{cmol}_{\mathrm{c}} \mathrm{kg}^{-1}\right)$ \\
\hline 1 & 19,0 \\
2 & 9,9 \\
3 & 7,5 \\
4 & 12,4 \\
5 & 2,9 \\
6 & 10,6 \\
\hline
\end{tabular}

dos cátions metálicos totais trocáveis $\left(\mathrm{Ca}^{+2}, \mathrm{Mg}^{+2}, \mathrm{~K}^{+}, \mathrm{Na}^{+}\right)+(\mathrm{H}+\mathrm{Al})$.

A determinação da CTC efetiva $\left(\mathrm{cmol} \mathrm{kg}^{-1}\right)$ nos sedimentos avaliados neste estudo é mostrada na Tabela 2 .

Valores de CTC maiores que $5 \mathrm{cmol}_{\mathrm{c}} \mathrm{kg}^{-1}$ evidenciam uma alta capacidade de troca de cátions ${ }^{35,40}$. Somente a amostra 5 não apresentou uma alta capacidade de troca de cátions. Os valores mais elevados para CTC nas amostras de sedimento ocorreram no ponto de amostragem 1.

\section{Determinações dos metais pseudo-totais e biodisponíveis}

Nas Tabelas 3 e 4 apresentam-se os resultados das concentrações $\left(\mathrm{mg} \mathrm{kg}^{-1}\right)$ dos metais pseudo-totais e dos metais biodisponíveis no sedimento, respectivamente.

Observa-se que foram encontradas concentrações de $\mathrm{Cu}, \mathrm{Zn}$ e $\mathrm{Pb}$ pseudo-totais e $\mathrm{Zn}$ e $\mathrm{Pb}$ biodisponíveis acima do valor máximo permitido, segundo a literatura ${ }^{41}$. Os pontos 1 e 2 apresentaram as maiores concentrações desses metais. No ponto 1 foi observada uma concentração de $\mathrm{Zn}, \mathrm{Pb}, \mathrm{Cu}$ pseudo-total de aproximadamente 38,57 e 3 vezes, respectivamente, maiores que os valores normalmente encontrados, segundo a literatura ${ }^{41}$. Já para as concen- trações biodisponíveis foram encontrados valores 36 e 40 vezes maiores para $\mathrm{Zn} \mathrm{e} \mathrm{Pb}$, respectivamente. No ponto 2 foram observadas concentrações de $\mathrm{Zn}$ e $\mathrm{Pb}$ pseudo-totais 10 e 14 vezes, respectivamente, maiores que os valores normalmente encontrados. Já para as concentrações biodisponíveis foram encontrados valores 6 e 4 vezes maiores para $\mathrm{Zn}$ e $\mathrm{Pb}$, respectivamente.

O PETAR foi sempre foco de interesse da indústria mineradora, pois a região do Vale do Ribeira comporta grandes depósitos de cobre, chumbo e zinco. As atividades de mineração, durante anos, geraram a contaminação da biota, devido às grandes concentrações de chumbo, zinco e cobre lá encontradas ${ }^{21-24}$. A mina de Furnas, localizada dentro do PETAR, possui mineralização associada a epicalcário dolomítico com intercalação de filitos por vezes grafitosos, com biotita e/ou clorita e quartzo ${ }^{21}$.

As amostras de sedimento coletadas no rio Furnas (ponto 1) e no rio Betari (ponto 2), logo após o rio Furnas, mostraram-se fortemente influenciadas pelos materiais de rejeito da mina de Furnas. Esse comportamento pode ser atribuído ao carreamento (lixiviados) de rejeitos de chumbo, zinco e cobre deixados a céu aberto próximo à mina abandonada, ao longo do percurso do rio. Contudo, a mina de Furnas desativada ainda influencia nas concentrações de chumbo, zinco e cobre ao longo do Rio Betari, que deságua no rio Ribeira de Iguape. Essas concentrações decrescem ao longo do rio até seu desemboque no mar, indicando que os metais são sedimentados ao longo do percurso ${ }^{21}$.

Os metais podem ser acumulados nos sedimentos de lagos e rios, e mudanças nas condições ambientais podem afetar sua biodisponibilidade. Uma vez sedimentados esses metais podem ser novamente redisponibilizados para a coluna d'água, graças a reações de oxi-redução, ou a processos de re-suspensão de origem física (correnteza), biológica (atividade dos organismos que vivem nos sedimentos) e humana (dragagem e navegação).

Tabela 3. Concentração $\left(\mathrm{mg} \mathrm{kg}^{-1}\right)$ dos metais pseudo-totais no sedimento nos diferentes pontos de amostragem

\begin{tabular}{|c|c|c|c|c|c|c|c|c|c|c|}
\hline \multirow{2}{*}{\multicolumn{2}{|c|}{$\begin{array}{l}\text { Pontos de } \\
\text { amostragem }\end{array}$}} & \multicolumn{9}{|c|}{ Concentração (mg kg-1) } \\
\hline & & $\mathrm{Zn}$ & $\mathrm{Cu}$ & $\mathrm{Cr}$ & $\mathrm{Mn}$ & $\mathrm{Fe}$ & $\mathrm{Ni}$ & $\mathrm{Cd}$ & $\mathrm{Pb}$ & Al \\
\hline 1 & \multicolumn{2}{|c|}{$5497,36 \pm 434,22$} & $103,95 \pm 2,04$ & $63,97 \pm 3,98$ & $2785,36 \pm 10,77$ & $53841,28 \pm 3053,16$ & $41,31 \pm 2,30^{*}$ & $10,00 \pm 0,0 *$ & $7569,78 \pm 439,72$ & $7989,67 \pm 747,04$ \\
\hline 2 & \multicolumn{2}{|c|}{$1412,29 \pm 63,91$} & $133,23 \pm 3,00$ & $37,30 \pm 2,28$ & $1205,80 \pm 120,79$ & $48231,18 \pm 1092,34$ & $31,98 \pm 1,98$ & $2,00 \pm 0,0 *$ & $1823,94 \pm 128,12 *$ & $14089,86 \pm 459,16$ \\
\hline 3 & \multicolumn{2}{|c|}{$416,50 \pm 15,29 *$} & $26,66 \pm 1,16$ & $29,32 \pm 2,32$ & $449,16 \pm 30,44$ & $23590,93 \pm 2168,38$ & $<\mathrm{LQ}$ & ND & $205,25 \pm 8,26^{*}$ & $13787,80 \pm 76,04$ \\
\hline 4 & \multicolumn{2}{|c|}{$1032,65 \pm 23,50$} & $95,27 \pm 7,60$ & $42,64 \pm 1,13$ & $421,05 \pm 37,40$ & $33777,58 \pm 2835,75$ & $27,32 \pm 2,31$ & ND & $117,25 \pm 3,03$ & $25723,07 \pm 1361,15$ \\
\hline 5 & \multicolumn{2}{|c|}{$329,96 \pm 0,08^{*}$} & $109,32 \pm 3,05$ & $30,00 \pm 2,00$ & $1159,86 \pm 72,35$ & $54725,42 \pm 4905,37$ & $28,00 \pm 1,99$ & ND & $115,98 \pm 5,28$ & $25223,30 \pm 1170,23$ \\
\hline 6 & \multicolumn{2}{|c|}{$639,83 \pm 9,74$} & $47,99 \pm 2,00 *$ & $34,66 \pm 1,16$ & $731,15 \pm 50,97$ & $32324,97 \pm 2537,12$ & $24,66 \pm 1,14$ & ND & $635,82 \pm 45,59 *$ & $18261,73 \pm 1172,41$ \\
\hline
\end{tabular}

Acima da concentração máxima permissível $(\mathrm{Cd}=30, \mathrm{Cr}=\mathbf{1 7 2 0}, \mathrm{Cu}=\mathbf{7 3}, \mathbf{P b}=\mathbf{4 8 0 0}, \mathbf{N i}=\mathbf{4 4}, \mathbf{Z n}=\mathbf{6 2 0})$, *acima da concentração negligenciável, em $\mathrm{mg} \mathrm{kg}^{-1}(\mathrm{Cd}=1,1, \mathrm{Cr}=116, \mathrm{Cu}=36, \mathrm{~Pb}=132, \mathrm{Ni}=35, \mathrm{Zn}=145)$, segundo a literatura ${ }^{41}$. $\mathrm{ND}=\mathrm{não}$ detectado, $\mathrm{LQ}=\mathrm{limite}$ de quantificação.

Tabela 4. Concentração $\left(\mathrm{mg} \mathrm{kg}^{-1}\right)$ dos metais biodisponíveis no sedimento nos diferentes pontos de amostragem

\begin{tabular}{|c|c|c|c|c|c|c|c|c|c|c|}
\hline \multirow{2}{*}{\multicolumn{2}{|c|}{$\begin{array}{l}\text { Pontos de } \\
\text { amostragem }\end{array}$}} & \multicolumn{9}{|c|}{ Concentração (mg kg $\left.{ }^{-1}\right)$} \\
\hline & & $\mathrm{Zn}$ & $\mathrm{Cu}$ & $\mathrm{Cr}$ & Mn & $\mathrm{Fe}$ & $\mathrm{Ni}$ & $\mathrm{Cd}$ & $\mathrm{Pb}$ & $\mathrm{Al}$ \\
\hline 1 & \multicolumn{2}{|c|}{$5247,27 \pm 451,71$} & $9,49 \pm 0,25$ & ND & $355,64 \pm 9.36$ & $478,91 \pm 16,01$ & ND & $4,50 \pm 0,00 *$ & $5280,57 \pm 118,87$ & $926,99 \pm 81,98$ \\
\hline 2 & \multicolumn{2}{|c|}{$802,47 \pm 21,24$} & $7,07 \pm 0,82$ & ND & $205,41 \pm 19.19$ & $863,92 \pm 15,61$ & $1,83 \pm 0,15$ & $1,25 \pm 0,00 *$ & $556,31 \pm 6,30^{*}$ & $641,12 \pm 2,29$ \\
\hline 3 & \multicolumn{2}{|c|}{$179,55 \pm 7,49 *$} & $5,24 \pm 0,02$ & ND & $286,02 \pm 27,11$ & $537,89 \pm 22,97$ & ND & ND & $100,92 \pm 1,25$ & $501,26 \pm 1,95$ \\
\hline 4 & \multicolumn{2}{|c|}{$69,05 \pm 4,00$} & $17,47 \pm 0,21$ & ND & $301,12 \pm 9,77$ & $2587,18 \pm 82,27$ & $3,08 \pm 0,30$ & ND & $42,59 \pm 0,39$ & $1033,94 \pm 14,19$ \\
\hline 5 & \multicolumn{2}{|c|}{$14,91 \pm 0,29$} & $3,50 \pm 0,25$ & ND & $178,28 \pm 18,79$ & $559,01 \pm 18,74$ & ND & ND & $21,66 \pm 0,88$ & $726,46 \pm 63,51$ \\
\hline 6 & \multicolumn{2}{|c|}{$515,75 \pm 15,71 *$} & $14,72 \pm 1,11$ & ND & $623,88 \pm 12,93$ & $826,83 \pm 7,04$ & $4,41 \pm 0,38$ & ND & $370,98 \pm 5,56^{*}$ & $752,05 \pm 56,59$ \\
\hline
\end{tabular}

Acima da concentração máxima permissível $(\mathrm{Cd}=30, \mathrm{Cr}=\mathbf{1 7 2 0}, \mathrm{Cu}=\mathbf{7 3}, \mathbf{P b}=\mathbf{4 8 0 0}, \mathbf{N i}=\mathbf{4 4}, \mathbf{Z n}=\mathbf{6 2 0})$, *acima da concentração negligenciável, em $\operatorname{mgkg}^{-1}(\mathrm{Cd}=1,1, \mathrm{Cr}=116, \mathrm{Cu}=36, \mathrm{~Pb}=132, \mathrm{Ni}=35, \mathrm{Zn}=145)$, segundo a literatura ${ }^{41} . \mathrm{ND}=$ não detectado. 
Os sedimentos avaliados neste estudo encontram-se altamente contaminados em alguns locais. O ambiente no momento encontra-se parcialmente controlado, graças às condições atuais, impedindo a solubilização dos metais para a água.

Deve-se lembrar que o chumbo é um metal ligado à poluição e tóxico, bioacumulativo e sem função biológica conhecida, tanto para as plantas como para os seres humanos. Formas orgânicas tóxicas deste metal estão também presentes no meio ambiente a partir de fontes diretas, além da possível metilação química/biológica de chumbo inorgânico em sedimentos anaeróbios ${ }^{42,43}$. Uma fração significativa de chumbo insolúvel pode ser incorporada em material particulado de superfície de escoamento, como íons sorvidos (adsorvidos e absorvidos) ou ainda na cobertura de superfície em sedimentos. A maior parte do chumbo é retida nos sedimentos e muito pouco é transportado em águas de superfície ou subterrâneas ${ }^{42,}{ }^{44}$.

No ambiente aquático o zinco prende-se, predominantemente, ao material suspenso antes de ser acumulado ao sedimento ${ }^{44}$. No entanto, a ressolubilização em fase aquosa é possível, sob certas condições físico-químicas, como na presença de ânions solúveis, na ausência de matéria orgânica, minerais de argila e hidróxidos de ferro e manganês, baixo $\mathrm{pH}$ e salinidade aumentada.

O cobre e o zinco são considerados elementos biologicamente essenciais, com papel enzimático em baixas concentrações, mas capazes de causar danos à saúde, se ingeridos em alta concentração.

\section{Correlação entre a CTC e as concentrações de metais biodisponíveis}

Os valores das concentrações de metais biodisponíveis $\left(\mathrm{mgkg}^{-1}\right)$ em sedimento são proporcionais à CTC $\left(\mathrm{cmol} \mathrm{kg}^{-1}\right)$ encontrada para essas mesmas amostras. As Figuras 2 e 3 apresentam a relação entre CTC e concentração biodisponível de cada metal, e CTC e a soma de todas as concentrações biodisponíveis, respectivamente.

Os sedimentos estão constituídos por diferentes substratos geoquímicos, os quais possuem alta superfície específica alta capacidade de troca catiônica ${ }^{7}$. Os mais importantes substratos são aqueles que possuem capacidade de reter e concentrar elementostraço. A característica fundamental desses materiais é a instabilidade termodinâmica, sendo constituídos por substâncias amorfas ou pouco cristalinas ${ }^{45}$. O ponto 1 mostrado neste estudo contém altas concentrações de metais e, como visto anteriormente, este mesmo ponto apresentou uma alta CTC em comparação aos demais pontos de amostragem, ressaltando que a disponibilidade dos metais nos sedimentos depende de quão forte é a interação ligante/ substrato geoquímico.

O papel significativo que os sedimentos desempenham nos ecossistemas aquáticos é bem conhecido. Além de fornecerem habitat para muitos organismos aquáticos, atuam também como fonte e depósito de materiais orgânicos e inorgânicos. De acordo com o tempo de residência na água do rio, pode ocorrer uma maior ou menor sedimentação dos materiais orgânicos e inorgânicos trazidos via entradas pontuais ou difusas. Deve-se, também, salientar a possibilidade de remoção e suspensão dos materiais orgânicos e inorgânicos nos sedimentos, devido ao aumento do fluxo de água ocasionado pelas chuvas, situação esta presente à época de coleta ${ }^{46}$.

O PETAR, por ser uma reserva regida por lei estadual de proteção ambiental, passa por uma série de conflitos, principalmente no que diz respeito à ocupação humana e ao turismo local. O turismo mantém a maior parte dos empregos no bairro, influenciando sua aceitação pela população, mesmo acarretando problemas, como a degradação ambiental ${ }^{16-20,31}$.

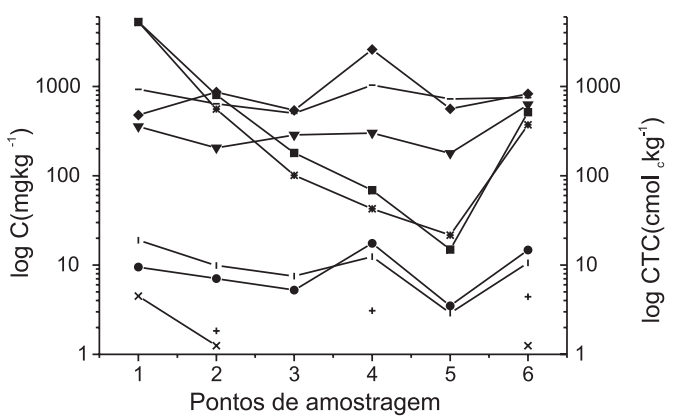

Figura 2. Gráfico da relação entre as concentrações dos metais biodisponíveis $\left(\mathrm{mgkg}^{-1}\right)$ e a CTC $\left(\mathrm{cmol}_{\mathrm{c}} \mathrm{kg}^{-1}\right)-\mathbf{-} \mathrm{Zn} ;-\bullet-\mathrm{Cu} ;-\mathbf{\Delta}-\mathrm{Cr} ;-\mathbf{\nabla}-$ $\mathrm{Mn} ;-\longrightarrow \mathrm{Fe} ;-+\mathrm{-}-\mathrm{Ni} ;-\times-\mathrm{Cd} ;-$ * $-\mathrm{Pb} ;---\mathrm{Al} ;-\mathrm{I}-\mathrm{CTC}$

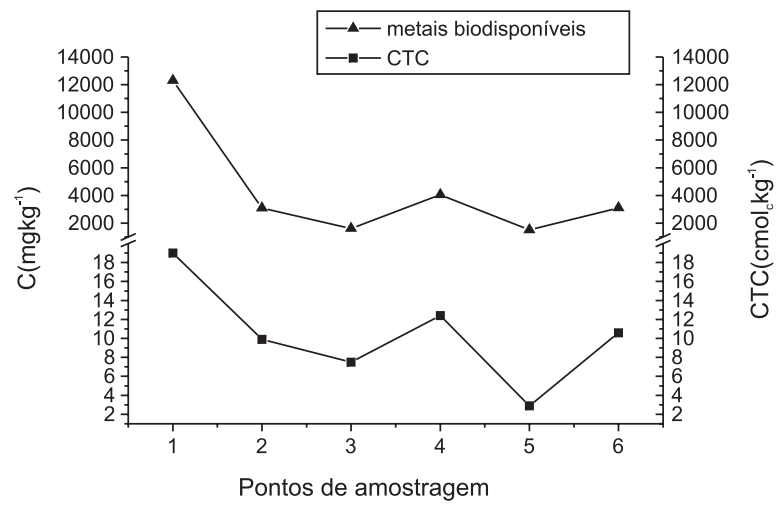

Figura 3. Gráfico da relação entre a soma das concentrações dos metais biodisponíveis $\left(\mathrm{mgkg}^{-1}\right)$ e a CTC $\left(\mathrm{cmol} \mathrm{kg}^{-1}\right)$

\section{CONCLUSÕES}

Os sedimentos coletados mostraram-se importantes compartimentos acumuladores de chumbo, zinco e cobre. As condições ambientais favorecem a ocorrência de formas precipitadas associadas ao sedimento. Dessa forma, o rio Betari funciona como transportador de íons para o rio Ribeira de Iguape.

A presença de rejeitos de mineração na sub-bacia do rio Furnas foi responsável pelos valores elevados de zinco, cobre e chumbo, observados no estudo, principalmente no ponto 1 (rio Furnas).

Outras minas abandonadas no local podem apresentar rejeitos expostos, como em Furnas, o que pode justificar as oscilações nas concentrações dos metais nos sedimentos ao longo do rio Betari.

As concentrações dos metais encontradas para sedimentos do PETAR refletiram a contribuição de origem natural, associada à geologia local, bem como a contribuição industrial mineradora que havia na região. No estudo pode-se observar que embora tenha cessado a exploração de chumbo, os efeitos ambientais dessa atividade continuam presentes.

\section{AGRADECIMENTOS}

Ao técnico do Departamento de Ecologia e Biologia Evolutiva da UFSCAR, Sr. A. S. Soares, pelas coletas. Ao laboratório de Estradas do Departamento de Transportes da Escola de Engenharia de São Carlos da Universidade de São Paulo, pelas análises granulométricas. Ao CNPq pela bolsa de mestrado concedida e à FAPESP pelo financiamento do projeto de Políticas Públicas, processo \# 01/12920-0. 


\section{REFERÊNCIAS}

1. Scorzelli, I. B.; Tese de Doutorado, Pontifícia Universidade Católica, Brasil, 1999.

2. Malavolta, E. ; Fertilizantes e seu Impacto Ambiental: metais pesados, mitos, mistificação e fatos, Produquímica: São Paulo, 1994.

3. Jordão, C. P.; Alves, N. M.; Pereira, J. L.; Bellato, C. R.; Alvares, V. H.; Quim. Nova 2000, 23, 5.

4. Mozeto, A. A.; Manejo da qualidade da água e da dinâmica do sedimento e do particulado da represa do Guarapiranga e do rio Grande, RMSP, UFSCAR: São Carlos, 1996. (Projeto RHAE, processo n0610419/95-1).

5. Lima, M. C.; Giacomelli, M. B. O.; Süpp, V.; Roberge, F. D.; Barrera, P. B; Quim. Nova 2001, 24, 734.

6. Jesus, H. C.; Costa, E. A.; Medonça, A. S. F.; Zandonade, E.; Quim. Nova 2004, 27, 378 .

7. Horowitz, A.; A primer on sediment- trace element chemistry, $2^{\text {nd }}$ ed., Lewis Publishers, 1991.

8. Santos, A.; Dissertação de Mestrado, Universidade de São Paulo, Brasil, 1999.

9. Perin, G.; Fabris, R.; Manente, S.; Rebello Wagener, A.; Hamacher, C.; Scotto, S.; Water Res. 1997, 31, 3017.

10. Audry, S.; Schäfer, J.; Blanc, G.; Jouanneau, J.; Environ. Pollut. 2004, 132, 413.

11. An, Y.; Kampbell, D. H.; Environ. Pollut. 2003, 122, 253.

12. Machado, W.; Moscatelli, M.; Rezende, L. G.; Lacerda, L. D.; Environ. Pollut. 2002, 120, 455.

13. Baptista Neto, J. A.; Smith, B. J.; McAllister, J. J.; Environ. Pollut. 2000 109, 1.

14. Secretaria do Meio Ambiente; Projeto PETAR - Parque Estadual Turistico do Alto Ribeira, SEMA: São Paulo, 1991. (Relatório Técnico).

15. http://www.unb.br/ig/sigep/sitio043/sitio043.pdf, acessada em Março 2005.

16. http://era.esa.chalmers.se/RosanaM/Publications/Rep\%20ERAPETAR\%2099.pdf, acessada em Março 2005.

17. Moraes, R.; Landis, W. G.; Molander, S.; Hum. Ecol. Risk Assess. 2002 $8,1779$.

18. Moraes, R.; Elfvendahl, S.; Kylin, H.; Molander S.; Ambio 2003, 32, 258.

19. Moraes, R.; Molander, S.; Hum. Ecol. Risk Assess. 2004, 10, 349.

20. Gerhard, P.; Moraes, R.; Molander, S.; Environ. Biol. Fishes 2004, 71, 321.

21. Moraes, R. P.; Dissertação de Mestrado, Universidade Estadual de Campinas, Brasil, 1997.

22. Sepe, J.; Dissertação de Mestrado, Universidade Estadual de Campinas, Brasil,1997.

23. Silva, R. H. P.; Dissertação de Mestrado, Universidade Estadual de Campinas, Brasil, 1997.

24. La Rosa, R. T.; Dissertação de Mestrado, Universidade Estadual de Campinas, Brasil, 1999.
25. Melcher, G. C.; Dissertação de Livre Docência, Universidade de São Paulo, Brasil, 1968.

26. Companhia de Tecnologia de Saneamento Ambiental; Avaliação da Qualidade Ambiental do Rio Ribeira do Iguape - Considerações Preliminares, CETESB: São Paulo, 1991. (Relatório Técnico).

27. Larsen, T. S.; Kristensen, J. A.; Asmund, G.; Bjerregaard, P.; Environ. Pollut. 2001, 114, 275

28. Shulkin, V. M.; Environ. Pollut. 1998, 101, 401.

29. Pagnanelli, F.; Moscardini, E.; Giuliano, V.; Toro, L.; Environ. Pollut. 2004, $132,189$.

30. Lacal, J.; Silva, M. P.; García, R.; Sevilla, M. T.; Procopio, J. R.; Hernández, L.; Environ. Pollut. 2003, 124, 291.

31. Cotta, J. A. O.; Dissertação de Mestrado, Universidade de São Paulo, Brasil, 2004.

32. Associação Brasileira de Normas Técnicas. Análise granulométrica de solos/método de ensaio, ABNT: Rio de Janeiro, 1982. (NBR 7181/1982).

33. Fiedler, H. D. L.; Tese de Doutorado, Universidade de Barcelona, Espanha, 1995.

34. Villaescusa-Celaya, J. A.; Gutiérrez-Galindo, E. A.; Flores-Munõz, G.; Environ. Pollut. 2000, 108, 453.

35. Jackson, M. L.; Soil Chemistry Analysis, India Privatte: New Delhi, 1967, p. 57.

36. Claessen, M. E. C.; Manual de métodos de análise do solo, 2a. ed., Embrapa: Rio de Janeiro, 1997.

37. Silva, F. C.; Manual de análises química de solos, plantas e fertilizantes, Embrapa: Brasília, 1999.

38. Mozeto, A. A.; Caracterização do estoque de metais pesados, compostos orgânicos voláteis e nutrientes dos sedimentos e dos particulados das represas do Guarapiranga e Billings, RMSP, Universidade Federal de São Carlos: Brasil, 1997. (Relatório final contrato FAI-UFSCAR/SABESP$\left.\mathrm{N}^{\circ} 012 / 97\right)$.

39. http://www.epa.gov/SW-846/pdfs/3050b.pdf, acessada em Março 2005

40. Van Raij, B.; Recomendações de adubação e calagem para o Estado de São Paulo, Instituto Agronômico, Fundação IAC: Campinas, 1996. (Boletim Técnico $\mathrm{n}^{0} 100$ ).

41. Crommentuijn, T. ; Sijm, D. ; Bruijn, J. ; Van Den Hoop, M. ; Van Leeuwen, K.; Van de Plassche, E.; J. Environ. Manag. 2000, 60, 121.

42. Sadiq, M.; Toxic metal chemistry in marine enviroments, Marcel Dekker: New York, 1992.

43. Forsyth, D. S.; Dabeka, R. W.; Cleroux, C.; Food Addit. Contam. 1991, 8, 477.

44. Alloway, B. J.; Heavy metals in soil, Blackie Academic and Professional: Glasgow, 1995.

45. Jones, B.; Bowser, C. Em Lakes: Chemistry, Geology, Physics; SpringerVerlag: New York, 1978, p. 179.

46. Tundisi, J. G.; Tundisi, T. ; Rocha, O. Em Águas doces no Brasil: capital ecológico, uso e conservação; Rebouças, A. C.; Braga, B.; Tundisi, J.G.; eds., Escrituras: São Paulo, 1999, p. 195. 